\title{
Multi-omics study of keystone species in the cystic fibrosis lung microbiome
}

Cynthia Silveira ( $\square$ cynthiabsilveira@gmail.com )

University of Miami https://orcid.org/0000-0002-9533-5588

Ana Georgina Cobian-Guemes

San Diego State University

\section{Carla Uranga}

J Craig Venter Institute

Jonathon Baker

J Craig Venter Institute

Anna Edlund

J Craig Venter Institute

Forest Rohwer

San Diego State University

Douglas Conrad

University of California San Diego

Research

Keywords: clindamycin, anaerobes, fermentation, mucus plugs, WinCF, metagenomics, metabolomics, transcriptomics

Posted Date: March 25th, 2020

DOI: https://doi.org/10.21203/rs.3.rs-18792/v1

License: (c) (1) This work is licensed under a Creative Commons Attribution 4.0 International License.

Read Full License 


\section{Multi-omics study of keystone species in the cystic fibrosis lung}

2 microbiome

3 Cynthia B. Silveira ${ }^{1,2,3}$, Ana G. Cobian-Guemes ${ }^{2,3}$, Carla Uranga ${ }^{4}$, Jonathon Baker ${ }^{4}$, Anna

$4 \quad$ Edlund $^{4}$, Forest Rohwer ${ }^{2,3}$ and Douglas Conrad ${ }^{5}$

5

$6{ }^{1}$ Department of Biology, University of Miami, 1303 Memorial Dr., FL 33146, USA.

$7 \quad{ }^{2}$ Department of Biology, San Diego State University, 5500 Campanile Dr., CA 92182, USA.

$8 \quad{ }^{3}$ Viral Information Institute, San Diego State University, 5500 Campanile Dr., CA 92182, USA.

$9 \quad{ }^{4}$ J. Craig Venter Institute, Genomic Medicine Group, La Jolla, CA, 92037, USA.

$10{ }^{5}$ Department of Medicine, Division of Pulmonary, Critical Care and Sleep Medicine, University

11 of California San Diego, La Jolla, California, USA.

12

13 cynthiasilveira@miami.edu, ana.naiboc@gmail.com, curanga@jcvi.org, jobaker@jcvi.org,

14 aedlund@jcvi.org, frohwer@gmail.com,dconrad@ucsd.edu

15

16 Correspondence to: cynthiasilveira@miami.edu

17

18 Kew words: clindamycin, anaerobes, fermentation, mucus plugs, WinCF, metagenomics, 19 metabolomics, transcriptomics 
Abstract

21 Background: Ecological networking and in vitro studies have predicted that anaerobic, mucus-

22 degrading bacteria are keystone species in cystic fibrosis (CF) microbiomes by sustaining the

23 growth of canonical CF pathogens. Here, a multi-omics approach was deployed to test this

24 hypothesis in vivo and in real time during a transition in antibiotic therapy of a CF patient with a

25 hypervariable lung function phenotype.

26 Results: Quantitative meta-omics and community culturing demonstrated that the use of a non-

27 traditional clindamycin therapy targeting gram-positives and gram-negative anaerobes re-

28 structured the entire CF microbial community. During rapid lung function loss, when the patient

29 was off antibiotics, the microbial community was dominated by anaerobic mucus-degrading

30 Streptococcus sp., Veilonella sp., and Prevotella sp. that produced fermentation gas and led to

31 the accumulation of fermentation products in sputum. The rise of anaerobes was followed within

326 days by an increase in Pseudomonas aeruginosa transcripts encoding the acquisition of

33 fermentation products from anaerobes and the production of virulence factors. The initiation of

34 clindamycin treatment reduced the fermentation and the abundance of anaerobes. Clindamycin

35 also lowered the abundance and transcription of P. aeruginosa, which is resistant to this

36 antibiotic. The treatment stabilized the patient's lung function and improved respiratory health

37 for two months, lengthening by a factor of four the between-hospitalization time for this patient.

38 Conclusions: The results presented here show that killing anaerobes, the weakest link in the

39 community in terms of antibiotic resistance, effectively limited the growth of classic CF

40 pathogen by disrupting community cross-feeding. The role that anaerobic, mucus-degrading

41 bacteria played in structuring the CF microbiome corroborates in vivo their position as keystone

42 bacteria, with high impact on community function despite lower relative abundances. 


\section{Introduction}

44 Keystone species are members of a community that have a disproportionately high importance in

45 ecosystem stability despite being less abundant than other community members [1]. In the

46 ecology of macro-organisms, these species commonly include top predators and first-level

47 consumers, which control ecosystem energy flow and, therefore, structure trophic relationships

$48[2,3]$. In the human microbiome, this ecological concept gave rise to the keystone-pathogen

49 hypothesis, which posits that "certain low-abundance microbial pathogens can orchestrate

50 inflammatory disease by remodeling a normally benign microbiota into a dysbiotic one" [4].

51 However, microbial ecology studies identify keystone species mainly based on their central

52 position in network analyses $[5,6]$. One caveat of this approach is that metabolic networks are

53 often built based on co-occurrence relationships in microbiomes (metagenomics) or their

54 activities (transcriptomics) [7, 8]. Identifying true keystone species require manipulative studies

55 of the microbiome $[9,10]$. Clinical treatment of polymicrobial diseases using narrow spectrum

56 antibiotics mimics this experimental approach and offers an invaluable opportunity to investigate

57 keystone species of the human microbiome in vivo.

58

59 Cystic fibrosis (CF) patients suffer from chronic polymicrobial infections throughout their lives

60 and are often treated with multiple antibiotics since early age [11]. Altered anion $\left(\mathrm{Cl}^{-}\right.$and

61 bicarbonate) transport in the CF respiratory epithelium result in decreased mucociliary clearance

62 and antimicrobial innate defenses, allowing the chronic colonization by a polymicrobial

63 community [12-15]. These communities elicit strong innate immune responses that drive the

64 airway wall remodeling and gas exchange abnormalities, eventually resulting in respiratory

65 failure or a need for lung transplantation in over $80 \%$ of $\mathrm{CF}$ patients. The polymicrobial nature 
66 of CF airway infections [11, 16-20] led to Climax-Attack ecological model the of the CF lung

67 microbiome [21]. The Attack community consists of transient members that elicit strong innate

68 immune responses and acute deterioration [22, 23]. In contrast, Climax communities cause

69 chronic infections and include most of the classic CF pathogens, including P. aeruginosa, which

70 are inherently resistant to anti-microbial therapy, grow slowly forming biofilms, and generate

71 suboxic environments in mucus plugs [24-26]. Hypoxic metabolisms are common in CF patients

72 with advanced lung disease or during exacerbations, when microbes use alternative electron

73 acceptors (nitrates, sulfates, iron, and fumarate) and fermentation [22, 27, 28], accumulating

74 toxic metabolic products in the lung [29, 30].

75

76 Previous network analysis and in vitro studies suggested that anaerobic bacteria function as

77 keystone species sustaining the microbial community and disease progression in $\mathrm{CF}[31,32]$.

78 The mechanistic basis of this hypothesis is the cross-feeding that occurs as mucin-degrading

79 anaerobes release fermentation products that sustain the growth of pathogens such as $P$.

80 aeruginosa, an inefficient mucus-degrading bacterium. Long-term 16S rRNA studies have also

81 shown an association between higher relative abundance of anaerobic bacteria including

82 Streptococcus sp. and Veillonella sp. with better health when P. aeruginosa is absent [33]. Here,

83 the onset of clindamycin treatment specifically targeting gram-positives and gram-negative

84 anaerobes during the clinical care of a CF patient offered an invaluable opportunity to test in vivo

85 the hypothesis that anaerobes are keystone species in CF. Quantitative multi-omics combined

86 with a community culture system showed that clindamycin suppressed not only anaerobes but

87 also $P$. aeruginosa. The disturbance of microbial cross-feeding improved the health of this

88 patient by extending the between-hospitalization time by a factor of four. 
Results

92 Clinical data: Patient 146 is part of the highly variable lung function group according to his

93 percent predicted Forced Expiratory Volume in 1 second (ppFEV1) [34]. In the year preceding

94 this study, the patient experienced 10 periods of rapid lung function loss that led to

95 hospitalization and intravenous (IV) antibiotic treatments (Supplementary Figure 1). The

96 ppFEV1 varied between 38 and $67 \%$ during that year (median $=53 \pm 5.7 \mathrm{SD})$. The clinical

97 microbiology tests were positive for P. aeruginosa, however the patient could not be stabilized

98 with oral antibiotic treatment after each hospital discharge. With the goal of identifying potential

99 causes for this hypervariable phenotype, a CF Rapid Response (CFRR) was launched at the time

100 of an exacerbation event in which the patient was admitted to the hospital for intravenous

101 antibiotic treatment [35]. During the Rapid response, metagenomes, transcriptomes and

102 metabolomes, community cultures and direct microbial counts were obtained from expectorated

103 sputum samples and analyzed in real-time (see Methods). At Day 0 (First day of CFRR), the

104 patient displayed acute decline in respiratory health characterized by severe cough, dyspnea and

105 a drop in his ppFEV1 to $44 \%, 9 \%$ below his median ppFEV1 (Figure S1). In the 27 days of

106 hospitalization that followed, the patient was treated with colistin, vancomycin, piperacillin-

107 tazobactam, and ceftazidime-avibactam, and the ppFEV1 increased to $59 \%$, leading to the

108 patient's discharge. This hospitalization period will be referred to as period A in this study.

109 During the following 8 days, named period B, he was off antibiotics. On day 33, six days after

110 hospital discharge, his ppFEV1 rapidly decreased to $50 \%$ and the patient complained of intense

111 cough and shortness of breath. Oral clindamycin treatment was initiated, and improved overall 
112 symptoms including cough and shortness of breath and maintained ppFEV1 at $49 \%$. This is

113 referred to as period C. The clinical microbiology tests were positive for P. aeruginosa before

114 CFRR, and a targeted search detected Rothia mucilaginosa in the clinical laboratory after it was

115 detected in the meta-omics data through CFRR (the dates of clinical microbiology tests are

116 shown in Figure S1). The patient was not under CFTR modulator treatment.

118 Microbial community structure: To investigate the community structure transitions across

119 changes in antibiotic regime and health status in Periods A, B and C, data from taxon-specific

120 microbial abundances, transcriptional activities, in vitro community culture (WinCF in Methods)

121 and total microbial abundances were analyzed by unsupervised random forest, a statistical

122 learning algorithm for identification of sample clusters and variables of importance. The random

123 forest identified three clusters of samples characterized by the patient's health status (Figure 1a

124 shows a 2D projection of the samples from a non-metric multidimensional scaling (NMDS)

125 analysis and clusters identified by the random forest are indicated by the dotted ellipses). The

126 first cluster includes samples from periods of lowest ppFEV1 and worst symptoms (cough and

127 shortness of breath) and coincides with highest WinCF fermentation and highest $P$. aeruginosa

128 abundance and transcriptional activity (cluster in the center of the NMDS plot in Figure 1a). The

129 antibiotic treatment in period A drove the community to a state of low microbial load and

130 correlated with the abundance and transcriptional activity of Rothia mucilaginosa (cluster on

131 right side of Figure 1a). As health deteriorated in period B, the abundance of Streptococcus,

132 Staphylococcus and Veillonella increased (also identified as cluster 2 in Figure 1a). This state

133 was followed by a return to low lung function with high fermentation, and high Pseudomonas

134 abundance and transcriptional activity (cluster 1 in the center Figure 1a). The onset of 
135 clindamycin treatment in period $\mathrm{C}$ drove the community to a third state, characterized by

136 Neisseria sp. and Haemophilus sp. abundance and transcriptional activity, with low total

137 microbial load and fermentation (cluster on the left side of Figure 1a). The random forest

138 indicated that the abundance of Streptococcus was the most important variable differentiating the

139 three clusters, followed by the abundance of Pseudomonas and Streptococcus transcriptional

140 ascitivies. A second random forest analysis supervised by the antibiotic regimes (periods A, B

141 and C) showed an out of bag error rate estimate of $11.1 \%$, in which Streptococcus abundance

142 was the variable with highest importance differentiating the periods, followed by Rothia,

143 Staphylococcus, and Haemophilus transcriptional activities (Figure 1b, p-values in the

144 permutational random forest test were $0.002,0.009,0.02$, and 0.04 , respectively).

146 The results of this multivariate statistical analysis led to the hypothesis that fermentation

147 products of mucus-degrading facultative anaerobes facilitated the rise of Pseudomonas

148 aeruginosa, a classical CF pathogen, through cross-feeding (Figure 1c). This mechanism of

149 interaction has been previously shown in vitro [32] and generated the following specific

150 predictions that could be tested with our dataset: 1- Facultative anaerobes, including

151 Streptococcus and Rothia, efficiently degrade mucus producing free-amino acids and short-chain

152 fatty acids (SCFA, i.e., propionate, acetate, butyrate, and butanediol). 2- The free amino acids

153 and SCFAs open a niche for the growth of Pseudomonas, which degrades mucins poorly.

154 Pseudomonas grows forming an anaerobic biofilm and produces phenazines. 3- The anoxic

155 biofilm created by Pseudomonas facilitates the growth of strict anaerobes, including Veillonella

156 and Prevotella. The whole microbial community grows, with Pseudomonas benefitting from the

157 metabolic products from anaerobes. 4- The onset of clindamycin treatment suppresses mucus- 
158 degrading anaerobes, destabilizing the community by the removal of the main nutritional source

159 for Pseudomonas, and decreasing total microbial abundances. How the meta-omics dataset met

160 the predictions of this working model is explored in detail in the following sections.

161

162 Bacterial abundances: Direct epifluorescence counts of bacterial cells were combined with

163 sputum metagenomes to quantify total bacterial load and taxon-specific abundances in cells per

$164 \mathrm{ml}$. The bacterial load in the sputum sample collected at Day 1 was $6.71 \times 10^{8}$ cells per ml, and

165 the community was dominated by Rothia mucilaginosa (78 \% of the community, Figure $2 \mathrm{a}$ ).

166 After 2 weeks of intravenous antibiotic in Period A, the bacterial load decreased to $2.9 \times 10^{7}$,

167 with most members of the community decreasing their absolute abundance, except for

168 Staphylococcus aureus and Veillonella dispar. In Period B, when the patient was off antibiotics,

169 the microbial abundance increased to $2.6 \times 10^{8}$. At this point, S. aureus and Streptococcus

170 sanguinis were the most abundant bacteria, followed by $R$. mucilaginosa and $P$. aeruginosa. The

171 clindamycin treatment (Period C) decreased microbial load to $2.4 \times 10^{7}$, and the community

172 became dominated by Haemophilus sp. and Neisseria sp. Both Haemophilus sp. and Neisseria

173 sp. decreased in abundance to less than $0.001 \%$ of the community after 2 months.

175 Bacterial transcriptional activity: Transcriptomes were mapped to the draft genome sequences

176 of Rothia mucilaginosa, Streptococcus sanguinis, Staphylococcus aureus, Veillonela dispar, and

177 Pseudomonas aeruginosa obtained from colonies isolated from the patient's sputum. The most

178 active community members were $R$. mucilaginosa, $S$. sanguinis, $V$. dispar, and $P$. aeruginosa,

179 respectively (Figure 2b). S. aureus was not among the most transcriptionally active, despite

180 representing up to $54 \%$ of the community three days post hospital discharge, according to 
181 metagenomic data. Antibiotic treatments changed the transcriptional activity: during intravenous

182 colistin, $R$. mucilaginosa was the most active member, followed by S. sanguinis and V. dispar.

183 Upon release from antibiotics, $V$. dispar transcripts increased, followed by $S$. sanguinis and $R$.

184 mucilaginosa. After six days off antibiotics, $P$. aeruginosa became the most active member of

185 the community, followed by $S$. sanguinis. Oral clindamycin treatment decreased transcript

186 abundance not only in the target species $S$. sanguinis, but also in $V$. dispar, $R$. mucilaginosa and

187 P. aeruginosa.

188

189 Fermentation: On Day 1, the WinCF community culture system produced the fermentation gas

190 that occupied $19 \%$ of the WinCF tube (Figure 3a). Simultaneously, the pH decreased in the tube

191 from 7.4 to below 5 starting at $0.33 \mathrm{~mm}$ (Figure $3 \mathrm{~b}$ ). The depth of tube where the $\mathrm{pH}$ changes

192 corresponds to the transition from the oxic to anoxic environment and is analogous to the oxygen

193 penetrance in the mucus plugs in the lung [29]. The amount of fermentation gas decreased to

194 non-detectable after 2 weeks of antibiotic treatment in period A (Figure 3a), and the depth of pH

195 change in the tubes increased (Figure 3b). After the hospital discharge, gas production increased

196 to $42 \%$ (Figure $3 \mathrm{a}$ ). This period corresponded to a decrease in $\mathrm{pH}$ change depth to $0 \mathrm{~mm}$ (Figure

$1973 \mathrm{~b}$ ), loss of lung function, and overall deterioration of his health. The patient initiated

198 clindamycin treatment 9 days after the hospital discharge, causing a sustained decrease in the

199 fermentation levels, down to to $4 \%$ after the first day of clindamycin and remaining below $3 \%$

200 for 60 days.

201

202 Metabolomes: The GC-MS metabolomic profiles showed that during the period the patient was 203 off antibiotics, there was an increase in the abundance of fermentation products (Figure 3c). 
204 Lactic acid, acetic acid and propanoic acid peaked 6 to 8 days after the patient stopped

205 intravenous colistin treatment and coincided with the increase in fermentation gas and

206 deterioration of heath (Figure 3a). There was also an increase in the abundance of free amino

207 acids and butaneidioic acid, a product of the metabolism of 1,4-butanediol.

208

209 Transcription of fermentation-related genes: The acetoin metabolism genes responsible for the

210 synthesis of the 2,3-butenediol and butanedione, a key metabolite in the cross-feeding between

211 Streptococcus and Pseudomonas, were transcriptionally active in patient CF146 [30]. The genes

212 encoding acetoin biosynthesis acetolactate synthase $(b u d B)$, acetolactate decarboxylase (budA),

213 and butanediol dehydrogenase (budC) were expressed by Streptococcus sp. and Staphylococcus

214 sp. at higher levels in periods of lung function loss (Figure 4a). Rothia also expressed budB early

215 in the sampling period, and Haemophilus and Neisseria expressed these genes after the onset of

216 clindamycin treatment. Pseudomonas aeruginosa was the main taxon expressing the genes aco $A$

217 and $a c o B$, whose products catabolize acetoin for utilization in central metabolism (Figure 4b).

218 The expression of aco genes was followed by the expression of phenazine by P. aeruginosa

219 (Figure 4b), a virulence factor that acts as alternative electron acceptor allowing $P$. aeruginosa

220 thrive in anoxic regions of biofilms and mucus plugs.

221

222 Discussion

223

224 Microbial colonization of the lungs in CF patients represents a case of complex microbial

225 dysbiosis driving disease progression, often without the identification of an individual causative

226 pathogen [25]. Metabolic networks have predicted that Streptoccoccus, Prevotella and 
227 Veillonella are the genera with the highest keystoneness in CF [31]. These species are efficient

228 mucus-degraders, and their anaerobic metabolism releases short-chain fatty acids that sustain the

229 growth of $P$. aeruginosa [32]. Our community-culture and multi-omics data show that these

230 pathways are active in the lungs of CF146. The growth of Streptococcus and Veillonella was

231 followed within two days by the growth of Rothia and Pseudomonas when the patient was off

232 antibiotics (Figures 3 and 4). The production of fermentation gas in the WinCF tubes and the

233 increase in abundance of short-chain fatty acids in the metabolomes demonstrates the activity of

234 fermenting bacteria and the release of metabolic by-products in sputum. Volatile fermentation

235 products such as lactic acid and 2,3-butanediol have been previously detected in CF breath gas

$236[30,36]$. These molecules can induce pyocyanine production, dormancy and biofilm formation in

237 P. aeruginosa, stimulating its growth and virulence [37, 38]. 2,3-butanediol has a direct toxic

238 effect on human cells [30]. The combined effects of fermentation products by direct attack to

239 host tissue and by stimulation of pathogen growth were the likely cause of the patient's lung

240 function decline in this study (ppFEV1 dropped from 59 to $50 \%$ six days post hospital

241 discharge).

242

243 The drop in lung function with an increase in anaerobic taxa and fermentation during period B

244 (no antibiotics) triggered the initiation of clindamycin treatment, a semisynthetic derivative of

245 lincomycin acting on ribosome translocation that inhibits protein synthesis in facultative gram-

246 positives such as Staphylococcus and Streptococcus, and anaerobic gram-negatives including

247 Bacteroides, Fusobacterium and Prevotella [39]. Clindamycin reduced the abundance and

248 activity of not only anaerobes, but also that of $P$. aeruginosa, which is resistant to this drug

249 (Figure 2). The decrease in Pseudomonas indicates its dependency on Streptococcus and 
250 Veillonela byproducts for growth. Pseudomonas's large genome is highly adapted to mucus

251 plugs, encoding alternate oxidative metabolism and virulence factors [40]. Yet, P. aeruginosa

252 grows poorly on mucins and depends on cross-feeding by mucus-fermenting anaerobes [32]. In

253 vitro antibiotic challenge targeting a mucin-fermenting community containing Veillonella

254 parvula, Fusarium nucleatum, Prevotella melaninogenica, and Streptococcus parasanguis in co-

255 cultures with $P$. aeruginosa controled both the fermenters and $P$. aeruginosa [41]. These

256 experiments demonstrated that the whole community minimum inhibitory concentration drops to

257 that of the weakest link - the least resistant species that provides resources to other community

258 members [41]. The dataset presented here provides evidence that this phenomenon occurs in vivo

259 and corroborates the role of Streptococcus and Veillonella as keystone genera in the CF

260 microbiome.

261

262 The conclusions presented here are based on an in-depth study of the lung disease progression in 263 one patient. Given the highly personalized nature of the CF microbiome, this pattern should be

264 tested in a larger cohort in the future. Previous studies with larger samples sizes based on 16S

265 rRNA data have shown that low diversity and dominance of a single pathogen are indicative of

266 rapid lung function loss [33]. This pattern is consistent with the concept of keystone species

267 presented here, where microbial community members with low abundance in the microbiome

268 have disproportionality high importance in community function, such as nutritionally sustaining

269 a dominant pathogen. While the multi-omics strategy presented here is still expensive for wide

270 deployment in the CF patient population, the WinCF cultures can be easily performed to indicate

271 fermentation, allowing selection of patients for in-depth studies. Regarding the non-traditional

272 application of clindamycin to treat CF lung infections, its long-term use can facilitate the 
273 development of colitis by Clostridium difficile colonization [42]. The suppression of keystone

274 species and metabolisms presented here offers a potential new focus for the the development of

275 novel strategies to manipulate the CF microbiome.

276

277 Conclusion

278

279 Here we show that a transition in antibiotic treatment of a CF patient re-structures the entire CF 280 microbiome by the suppression of keystone species that nutritionally sustained the growth of a 281 pathogen. Clindamycin therapy targeting anaerobes reduced fermentation and the abundance of

282 both anaerobes and $P$. aeruginosa, which is resistant to this antibiotic. The treatment improved

283 respiratory health for 2 months, lengthening by a factor of four the between-hospitalization time

284 for this patient. These results corroborate in vivo the hypothesis that anaerobes are keystone

285 members of the CF microbiome providing structure and stability to the whole microbial

286 community through cross-feeding.

287

288 Methods

289

290 Clinical data: Sample collection procedures and access to clinical data were approved by the 291 institutional review boards (IRBs) of the University of California San Diego (UCSD) (HRPP

292 081510), and San Diego State University (IRB approval number 1711018R). Clinical

293 microbiology and spirometry tests were performed for clinical indications during the normal care

294 of the patient. Spirometry tests were used to calculate the percentage of predicted FEV1 (forced

295 expiratory volume in one second), as previously described [43]. The age*FEV $1 \%$ predicted 
296 product is a derived variable calculated by multiplying the age of the individual at the most

297 recent FEV1 by the best FEV1\% during the previous year and is used to assess disease risk [34,

298 44].

299

WinCF community culture: Winogradsky CF community culture was modified from Quinn et al

3012015 by adjusting the $\mathrm{pH} 7.4$ prior to use in WinCF experiments [29, 45-47]. Briefly, sputum

302 samples were collected by expectoration, homogenized, and diluted 10-fold into sterile ASM.

303 The mixture was inoculated into capillary tubes that were plugged at one end and then laid

304 horizontally in a $100 \%$ humidity environment at $37^{\circ} \mathrm{C}$. At the end of incubations, capillary tubes

305 were imaged in the dark on white backlight (Logan $5.5 \mathrm{X} 9$ inches $^{2}$ light box) using a Canon

306 EOS Rebel T3 camera (Canon U.S.S Inc., Melville, NY, USA). All images were taken under

307 identical settings (manual focus, ISO 3,200, Aperture F4.5) and saved in raw and JPEG format.

308 Gas production was quantified as the percentage of capillary tube length occupied by gas. The air

309 penetrance in the capillary was defined as depth of the tube where $\mathrm{pH}$ dropped below 5 , as

310 indicated by color change in the phenol red/bromocresol purple tubes ( $\mathrm{pH}$ drops due to

311 fermentation products).

313 Metagenomes: Total DNA was extracted from sputum samples using an adapted PowerSoil

314 DNA Isolation kit protocol (Qiagen, Germany). The samples were subject to 5 freeze-thaw

315 cycles $\left(5\right.$ minutes flash-freeze, 5 minutes at $100^{\circ} \mathrm{C}$ ) followed by bead-beating for 45 minutes

316 using the bead tubes from the Power Soil kit. The DNA was eluted in molecular-grade water and

317 metagenome libraries were constructed using a Nextera DNA library preparation kit (Illumina,

318 CA). Libraries were sequenced on an Illumina MiSeq platform. Quality filtering and 
319 dereplication were done using PRINSEQ++ [48] with minimum quality threshold 20,

320 dereplication and entropy threshold 50. Cloning vector sequences were removed using SMALT

321 [49] with $80 \%$ identity against the NCBI UniVec database. Human genome sequences were

322 removed using SMALT with $80 \%$ identity against the human reference genome GRCh38.

323 Microbial taxonomy assignments at the genus level were made from SMALT a with $96 \%$

324 identity against the NCBI RefSeq database of complete bacterial genomes.

325

326 Bacterial isolation and genome sequencing: Fresh sputum sample from the first day of

327 hospitalization in this study (Day 0) were resuspended in saline buffer and plated on BHI agar.

328 Plates were incubated overnight at $37^{\circ} \mathrm{C}$. Colonies with distinct morphologies were picked and

329 re-plated on BHI plates. The five species that represented the most abundant members in the

330 metagenomes (by sequence recruitment at 96\% identity) were selected for full genome

331 sequencing: Rothia mucilaginosa, Streptococcus sanguinis, Staphylococcus aureus, Veillonela

332 dispar and Pseudomonas aeruginosa. Total DNA was extracted and prepared for sequencing

333 using KAPA HyperPlus (KAPA Biosystems, IN). The libraries were sequenced on an Illumina

334 MiSeq platform. Quality filtering, dereplication and cloning vector removal were performed as

335 above. Sequences were assembled using Spades [50].

336

337 Metabolomics: Sputum samples $(1 \mathrm{ml})$ were lyophilized before extraction to concentrate all

338 metabolites. Samples were extracted and subjected to fast chemical derivatization reaction using

339 methyl chloroformate [51]. Samples were analyzed by gas chromatography-mass spectrometry

340 (GC-MS). The data was analyzed using Automated Mass Deconvolution and Identification

341 System (AMDIS). 
343 Metatranscriptomics: Total RNA was extracted from raw sputum samples using guanidinium

344 thiocyanate according to manufacter's protocol (TRIzol, Invitrogen, CA). RNA was resuspended

345 in $50 \mu 1$ of RNAse-free water and DNase treatment was performed by adding $2 \mu 1$ of TURBO

346 DNase (Thermo Fischer Scientific, CA). First strand RNA was synthesized using Superscript II

347 reverse transcriptase (Invitrogen, CA). Sequencing libraries were prepared using the Ovation

348 Complete Prokaryotic RNA-Seq System (NuGEN Technologies, CA). Libraries were sequenced

349 in an Illumina HiSeq Platform. Quality filtering, dereplication and cloning vector removal were

350 performed as above. The transcripts were mapped to the NCBI RefSeq database of complete

351 bacterial genomes using SMALT at $96 \%$ identity. The number of transcripts mapping to a

352 genome was normalized by genome size using the FRAP to calculate relative number of

353 transcripts mapping to a given taxon within a sample. This relative transcriptional activity was

354 then multiplied by the number of cells per ml of sputum sample to obtain the taxon-specific

355 transcriptional activity per $\mathrm{ml}$ of sputum and allow between-sample comparison. This scaling

356 assumes that the total bacterial transcriptional activity per $\mathrm{ml}$ of sputum is proportional to the

357 bacterial abundance per $\mathrm{ml}$ of sputum. We also mapped the transcripts to the genomes of

358 bacterial isolates obtained from the same patient at $96 \%$ identity using SMALT. The relative

359 transcript abundance was normalized by absolute microbial abundances per ml of sample to

360 allow between-sample comparisons. The transcript recruitment to the isolates genomes was

361 visualized using ANVI'O [52].

362

363 Multivariate analyses: The absolute abundance of each genus of bacteria per ml was calculated

364 as the product of the fractional abundance in the metagenome [53] and the total microbial 
365 abundances determined by direct epifluorescence counts. The absolute abundances were

366 combined with taxon-specific transcriptomic activity per ml, WinCF fermentation and total

367 microbial abundances in one dataset (Supplementary Data 1) and analyzed through a non-metric

368 multi-dimensional scaling (NMDS) using Bray-Curtis distances in the vegan package in R. The

369 two first dimensions are visualized in 2D in Figure 1A. Samples were clustered using the

370 proximity matrix from an unsupervised random forest in the R package randomForest using 5000

371 trees and Ward method for hierarchical clustering. Random forest is a robust non-parametric,

372 statistical learning method for the identification of clusters and variables of importance in

373 complex multi-omics data $[54,55]$. The effect of different antibiotic regimes (clindamycin, no

374 antibiotics and the combination of colistin, piperacillin-tazobactam, ceftazidime-avibactam, and

375 vancomycin) on was tested using a supervised permutational random Forest (5000 trees 1000

376 permutations) where the three antibiotic regimes were used as supervising variable in the

377 package rfPermute. 


\section{Declarations}

379

380

Ethics approval and consent to participate

381 Sample collection procedures and access to clinical data were approved by the institutional

382 review boards (IRBs) of the University of California San Diego (UCSD) (HRPP 081510) and

383 San Diego State University (IRB approval number 1711018R).

384

385 Consent for publication

386 No identifiable data is included in this manuscript and this study was approved by the

387 institutional review boards of the University of California San Diego (UCSD) (HRPP 081510)

388 and San Diego State University (IRB approval number 1711018R)..

389

390 Availability of data and materials

391 Metagenomic data is available in the NCBI Short Read Archive (SRA) under BioProject ID

392 PRJNA580503. Genome sequences of clinical isolates are provided as contigs and associated

393 annotation files in the Pathosystems Resource Integration Center (PATRIC) server in the public

394 workspace csilveira / CF146. Transcriptomic data are available through the Gene Expression

395 Omnibus (GEO) server in NCBI record GSE139943. Codes used for statistical analysis are

396 provided in github under cbsilveira/CF146 and csv files used as input are provided as Additional

397 Data along with the manuscript.

398

399 Competing Interests

400 The authors declare no competing interests. 


\section{Funding}

403 This research was funded by the Cystic Fibrosis Research Inc. (S00022215 to CS and

404 S00023377 to FR) and Spruance Foundation.

405

406 Authors' contributions

407 CBS, FR and DC designed the study; CBS, AGCG, CU, JB and AE performed experiments,

408 analyses; CS wrote the manuscript and all authors contributed with revisions.

409

410 Acknowledgements

411 The authors thank Ryan Hunter, Stephanie Widder and Katrine Whiteson for discussions and

412 criticisms on cross-feeding, keystone species, and metabolomic data that significantly improved

413 this manuscript. 


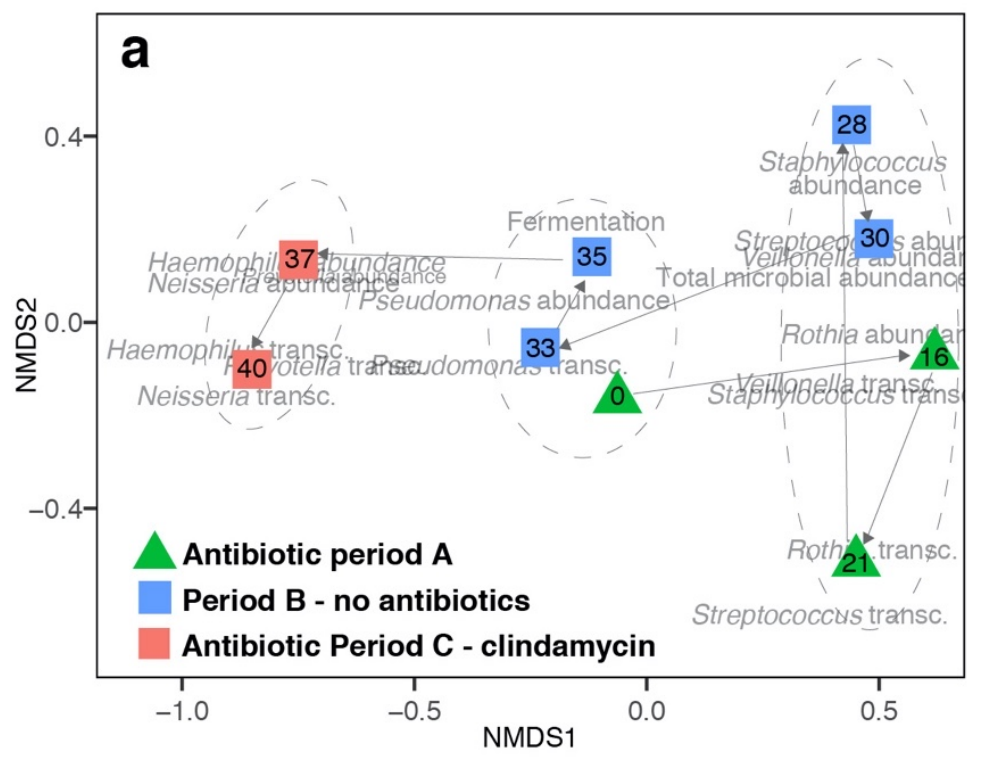

C

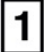

\section{2} mucus degradation

Streptococcus Rothia

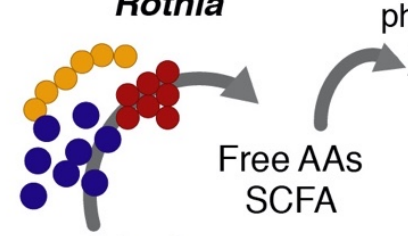

Mucins

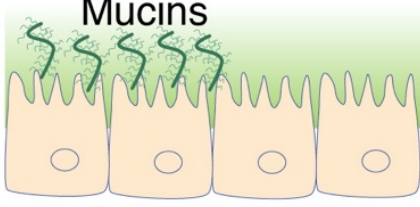

b

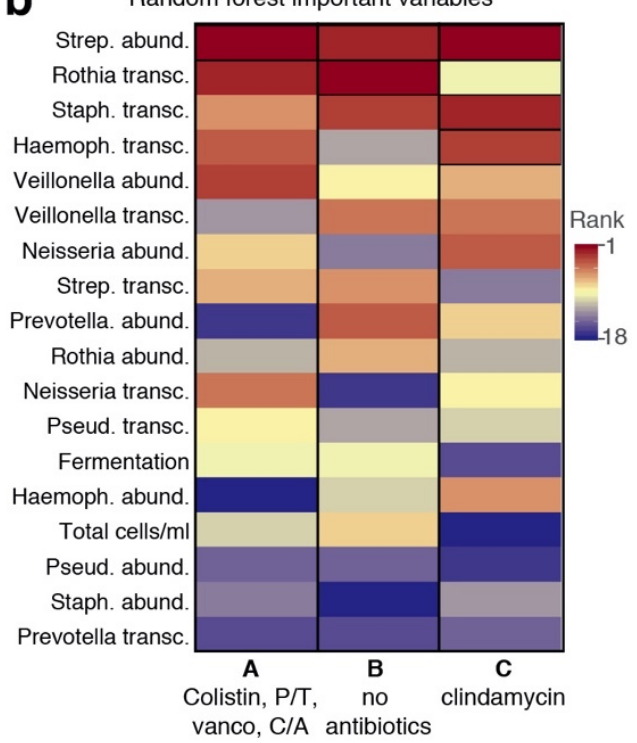

3

Anaerobic growth, mucus degradation, cross-feeding Veillonella Prevotella Streptococcus Staphylococcus Rothia mucus-degrading, Pseudomonas anaerobic biofilm

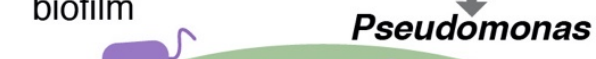

4

\section{Clindamycin}

Suppression of anaerobic, mucus-degrading bacteria leads to Pseudomonas starvation

417 Figure 1. Microbial community succession. a) Non-metric multidimensional scaling (NMDS)

418 of sputum samples over time. The variables used as input for the NMDS were WinCF

419 fermentation, total microbial abundances, genera-specific microbial abundances, and genera-

420 specific transcriptomic activity. Symbols are color-coded by antibiotic treatment periods (green:

421 A - colistin, vancomycin, ceftazidime-avibactam, and piperacillin-tazobactam; blue: B - no 
422 antibiotics; pink: C - clindamycin), and numbers inside the symbols indicate days since hospital

423 admission event when Rapid Response was initiated. The dotted ellipses indicate health status

424 groups supported by an unsupervised random forest analysis followed by clustering using Ward

425 distances (out of bag error $=11.1 \%$ ). b) Important variables differentiating treatment periods in

426 a classification random forest analysis supervised by treatments. The ranks surrounded by a

427 black box indicate variables with p-values less than 0.05 in the permutational test. c) Conceptual

428 model of succession events in patient CF146: 1- The mucosal surfaces are colonized by

429 facultative anaerobes, including Streptococcus and Rothia, capable of efficient mucus

430 degradation producing free-amino acids and short-chain fatty acids (SCFA, i.e., propionate,

431 acetate, butyrate, and butanediol). 2- The free amino acids and SCFAs open a niche for the

432 growth of Pseudomonas, which degrades mucins poorly. Pseudomonas grows forming an

433 anaerobic biofilm, and produces phenazines. 3- The Pseudomonas biofilm facilitates the growth

434 of anaerobes, including obligate anaerobes such as Veillonella and Prevotella. There is an overall 435 growth of the whole microbial community, with Pseudomonas benefitting from the metabolic

436 products from anaerobes. 4- The onset of clindamycin treatment suppresses the growth of gram-

437 positive anaerobes, such as Streptococcus and Staphylococcus. The suppression of mucus-

438 degrading bacteria removes the main nutritional source for Pseudomonas, leading to an overall

439 decrease in microbial abundances. Period A corresponds to 28 days of hospitalization when the

440 patient was treatment with colistin, vancomycin, piperacillin-tazobactam, and ceftazidime-

441 avibactam; in period Period B the patient was released and was off antibiotics; Period C is the

442 clindamycin treatment. 
a Microbial abundances per $\mathrm{ml}$ of sputum at genus level

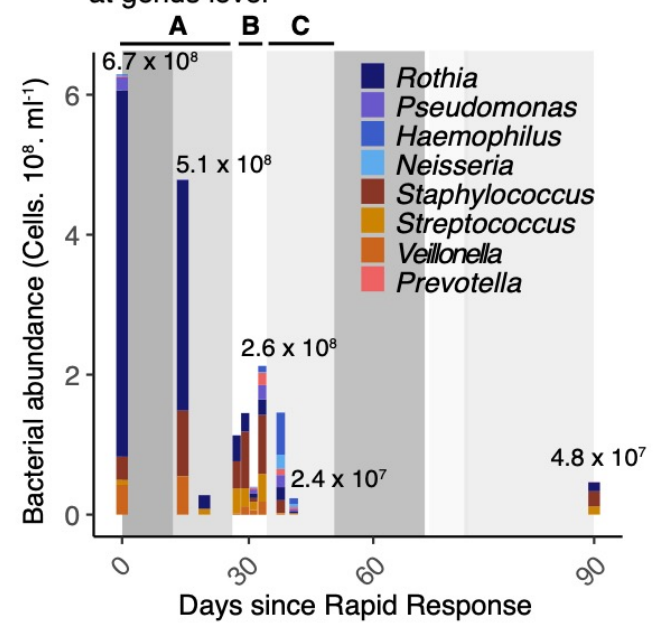

b Transcript abundace per $\mathrm{ml}$ of sputum at species level

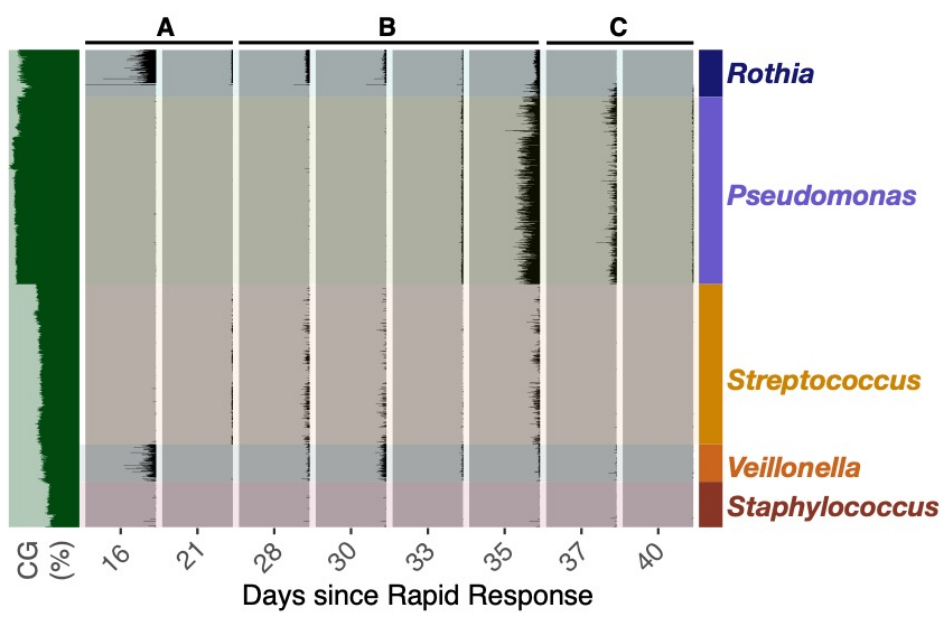

444 Figure 2. a) Changes in abundance of most abundant bacterial genera in the sputum from patient

445 CF146. Absolute abundances (in cell counts per $\mathrm{ml}$ of sputum) were obtained by multiplying the

446 fractional abundance from metagenomes by the total cell counts from epifluorescence

447 microscopy. b) Recruitment of metatranscriptomic reads to contigs obtained from sequencing the

448 genomes of clinical bacterial isolates. The height of each peak in the plot denotes mean coverage

449 of a contig divided by overall sample mean coverage and scaled to the absolute microbial

450 abundances per $\mathrm{ml}$ of sputum sample. As these are draft and not complete genomes, the position

451 of each contig along the $\mathrm{y}$ axis is defined by $\mathrm{k}-\mathrm{mer}$ similarity $(\mathrm{k}=4)$ rather than position in the

452 genome. The letters A, B and C within the plot area indicate the different antibiotic treatment

453 periods. 
a Fermentation in WinCF

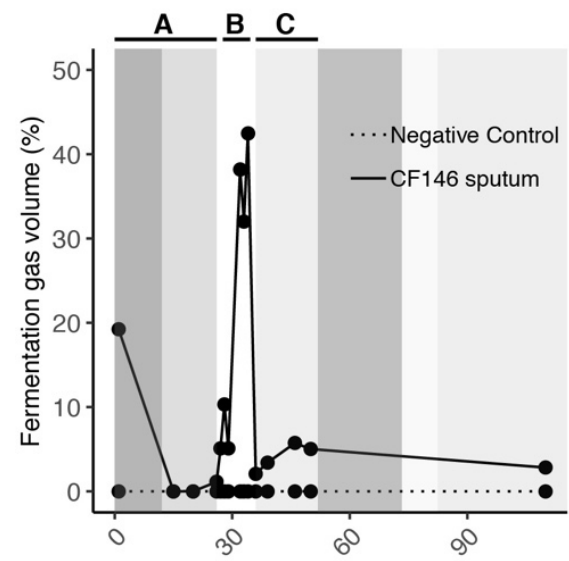

b Oxic layer in WinCF

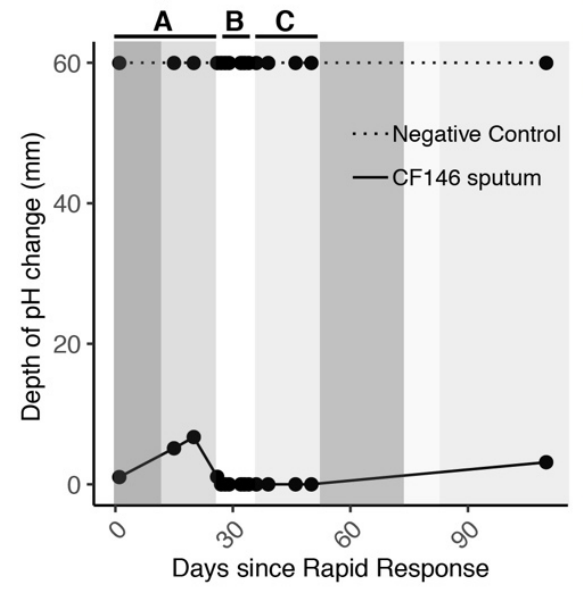

C GC metabolomics in sputum

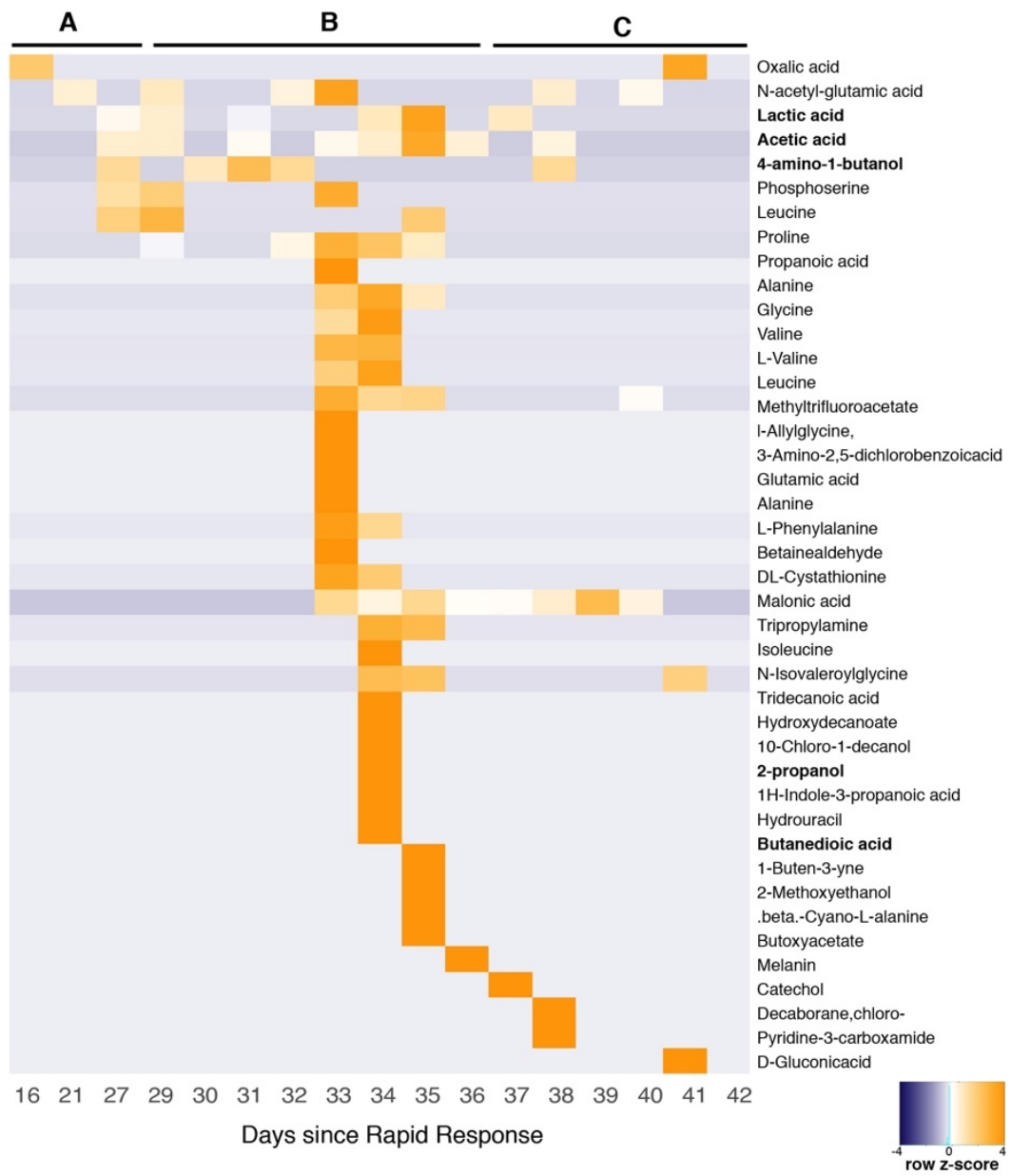

Figure 3. Fermentation in the sputum of patient CF146. a) production of fermentation gas in in

458 WinCF capillary tubes inoculated with sputum microbial communities in artificial sputum

459 media; b) depth of $\mathrm{pH}$ change in WinCF capillary tubes. The depth of tube where the $\mathrm{pH}$ changes

460 corresponds to the transition from the oxic to anoxic environment and is analogous to the oxygen

461 penetrance in the mucus plugs in the lung [29]. The letters A, B and C within the plot area

462 indicate the different antibiotic treatment periods. 
C Transcript abundance of acetoin and butanediol production genes

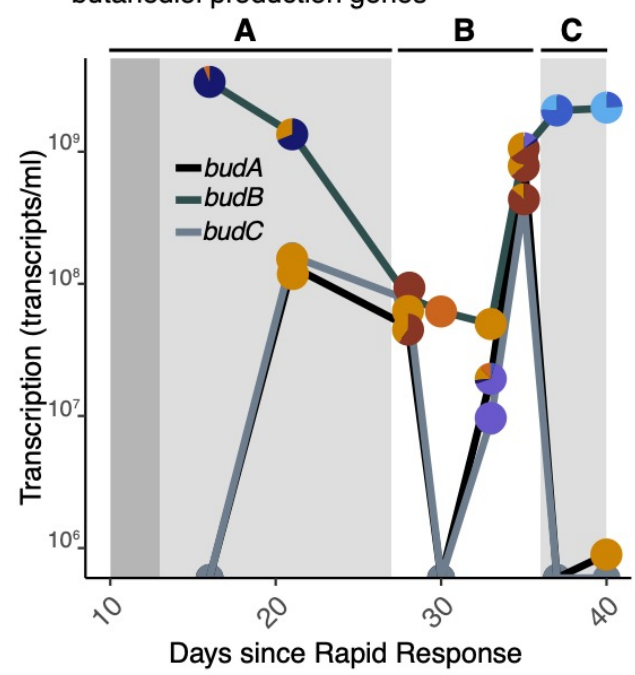

d Transcrip abundance of butanediol consumption and phenazine synthesis genes

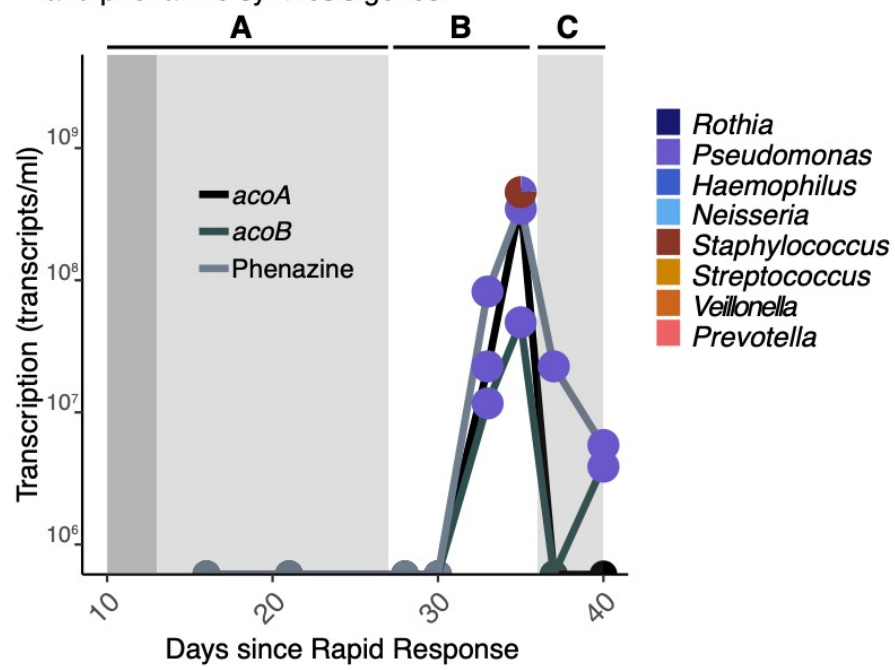

466 Figure 4. Microbial transcriptome dynamics. a) Abundance of transcripts involved in the

467 pathway of acetoin and butanediol production (budA, budB, and budC). Each point in the plot is

468 divided by the taxonomic assignment of the transcripts. The transcript abundance per ml (y axis)

469 was calculated as the product of the number of transcripts per $\mathrm{Kb}$ of a given taxon and the total

470 number of $\mathrm{Kbs}$ of a that same taxon, calculated from the absolute abundances and genome sizes.

471 b) Abundance of transcripts involved in the pathway of acetoin and butanediol consumption

$472(a c o A$ and $a c o B)$ and phenazine production ( $p h z A B C D E F G$, shown as a sum of all transcripts in

473 this gene cluster). Each point in the plot is divided by the taxonomic assignment of the

474 transcripts. The transcript abundance per ml (y axis) was calculated as in 4B.

475

476

477 


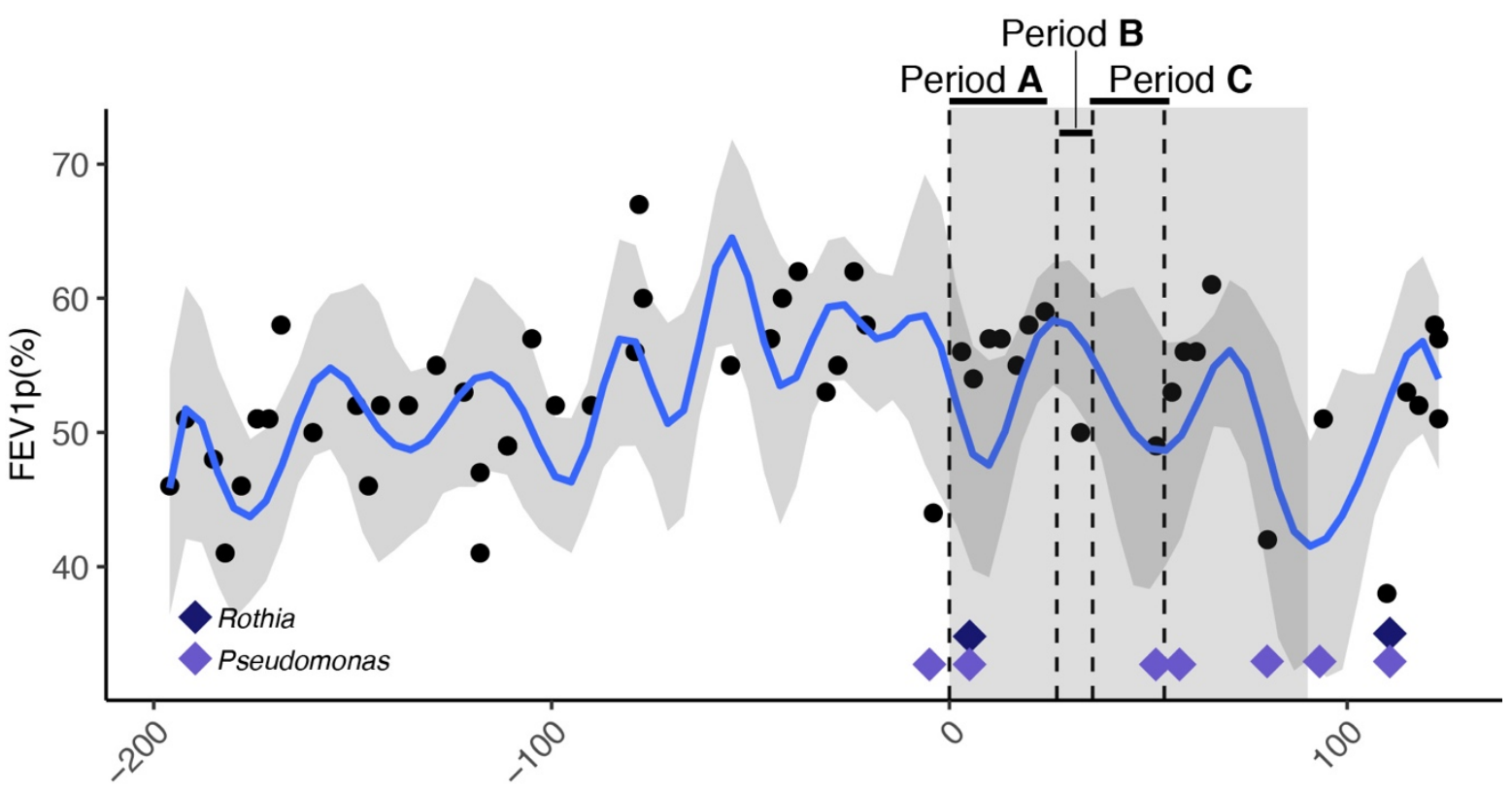

Days since hospital admission

481 Figure S1. Patient's lung function over time. Day zero indicates the first day of hospitalization

482 in the period focused on in this study. Percent predicted FEV1 (forced expiratory volume in one

483 second) are shown by the dots; the blue line shows the median spline regression (effective

484 degrees of freedom $=29$ ). The shadow block indicates the window of time analyzed here by

485 multi-omics. The diamond symbols indicate clinical microbiology tests positive for

486 Pseudomonas aeruginosa and Rothia sp. during stand care. The vertical dotted lines indicate the

487 three periods of interest in this study: Period A corresponds to 28 days of hospitalization when

488 the patient was treatment with colistin, vancomycin, piperacillin-tazobactam, and ceftazidime-

489 avibactam; in period Period B the patient was released and was off antibiotics; Period C is the

490 clindamycin treatment.

491

492 


\section{References}

494 1. Power ME, Tilman D, Estes JA, Menge BA, Bond WJ, Mills LS, et al. Challenges in the quest

495 for keystones. Bioscience. 1996; September.

496 2. Cortés-Avizanda A, Colomer MÀ, Margalida A, Ceballos O, Donázar JA. Modeling the

497 consequences of the demise and potential recovery of a keystone-species: wild rabbits and avian

498 scavengers in Mediterranean landscapes. Sci Rep. 2015;2015 November:1-12.

499 doi:10.1038/srep17033.

500 3. Pringle RM, Kartzinel TR, Palmer TM, Thurman TJ, Fox-dobbs K, Xu CCY, et al. Predator-

501 induced collapse of niche structure and species coexistence. Nature. 2019. doi:10.1038/s41586-

$502 \quad 019-1264-6$.

503 4. Hajishengallis G, Darveau RP, Curtis MA. The keystone-pathogen hypothesis. Nat Rev

504 Microbiol. 2012;10:717-25. doi:10.1038/nrmicro2873.

505 5. Banerjee S, Schlaeppi K, Heijden MGA. functioning. Nat Rev Microbiol. 2018.

506 doi:10.1038/s41579-018-0024-1.

507 6. Trosvik P, Jacques De Muinck E. Ecology of bacteria in the human gastrointestinal tract-

508 identification of keystone and foundation taxa. 2015.

509 7. Röttjers L, Faust K. Can we predict keystones? 2019;17 march:15007.

510 8. Roume H, Heintz-buschart A, Muller EEL, May P, Satagopam VP, Laczny CC, et al.

511 Comparative integrated omics : identi fi cation of key functionalities in microbial community-

512 wide metabolic networks. 2015; April.

513 9. Banerjee S, Schlaeppi K, Heijden MGA Van Der, Microbiol NR. Reply to ' Can we predict

514 microbial keystones ?' Nat Rev Microbiol. 2019;17 march:2019. doi:10.1038/s41579-018-0133-

$515 \mathrm{x}$. 
516 10. Lawson CE, Harcombe WR, Hatzenpichler R. Common principles and best practices for 517 engineering microbiomes. Nat Rev Microbiol. 2019.

518 11. Harris JK, De Groote MA, Sagel SD, Zemanick ET, Kapsner R, Penvari C, et al. Molecular 519 identification of bacteria in bronchoalveolar lavage fluid from children with cystic fibrosis. Proc 520 Natl Acad Sci. 2007;104:20529-33.

521 12. Quinton PM. Cystic fibrosis: impaired bicarbonate secretion and mucoviscidosis. Lancet. $522 \quad 2008 ; 372: 415-7$.

523 13. Alaiwa MHA, Reznikov LR, Gansemer ND, Sheets KA, Horswill AR, Stoltz DA, et al. pH 524 modulates the activity and synergism of the airway surface liquid antimicrobials $\beta$-defensin-3 525 and LL-37. Proc Natl Acad Sci. 2014;111:18703-8.

526 14. Kunzelmann K, Schreiber R, Hadorn HB. Bicarbonate in cystic fibrosis. J Cyst Fibros. $527 \quad 2017 ; 16: 653-62$.

528 15. Gibson RL, Burns JL, Ramsey BW. Pathophysiology and management of pulmonary 529 infections in cystic fibrosis. Am J Respir Crit Care Med. 2003;168:918-51.

530 16. Sibley CD, Parkins MD, Rabin HR, Duan K, Norgaard JC, Surette MG. A polymicrobial 531 perspective of pulmonary infections exposes an enigmatic pathogen in Cystic Fibrosis patients.

532 Proc Natl Acad Sci. 2008;105:15070-5.

533 17. Harrison F. Microbial ecology of the cystic fibrosis lung. Microbiology. 2007;153:917-23.

534 18. Tunney MM, Field TR, Moriarty TF, Patrick S, Doering G, Muhlebach MS, et al. Detection 535 of anaerobic bacteria in high numbers in sputum from patients with cystic fibrosis. Am J Respir 536 Crit Care Med. 2008;177:995-1001.

537 19. Lynch S V, Bruce KD. The cystic fibrosis airway microbiome. Cold Spring Harb Perspect 538 Med. 2013;3:a009738. 
539 20. Rogers GB, Carroll MP, Serisier DJ, Hockey PM, Jones G, Bruce KD. Characterization of

540 bacterial community diversity in cystic fibrosis lung infections by use of 16S ribosomal DNA

541 terminal restriction fragment length polymorphism profiling. J Clin Microbiol. 2004;42:5176-83.

542 21. Conrad D, Haynes M, Salamon P, Rainey PB, Youle M, Rohwer F. Cystic fibrosis therapy: a

543 community ecology perspective. Am J Respir Cell Mol Biol. 2013;48:150-6.

544 22. Whiteson KL, Bailey B, Bergkessel M, Conrad D, Delhaes L, Felts B, et al. The upper

545 respiratory tract as a microbial source for pulmonary infections in Cystic Fibrosis: parallels from

546 island biogeography. Am J Respir Crit Care Med. 2014;189:1309-15.

547 23. O’Toole GA. Cystic fibrosis airway microbiome: Overturning the old, opening the way for

548 the new. J Bacteriol. 2018;200:e00561-17.

549 24. Lim YW, Schmieder R, Haynes M, Willner D, Furlan M, Youle M, et al. Metagenomics and 550 metatranscriptomics: Windows on CF-associated viral and microbial communities. J Cyst Fibros. $551 \quad 2013 ; 12: 154-64$. doi:10.1016/j.jcf.2012.07.009.

552 25. Lim YW, Evangelista JS, Schmieder R, Bailey B, Haynes M, Furlan M, et al. Clinical

553 insights from metagenomic analysis of sputum samples from patients with cystic fibrosis. J Clin

554 Microbiol. 2014;52:425-37.

555 26. Cowley ES, Kopf SH, LaRiviere A, Ziebis W, Newman DK. Pediatric cystic fibrosis sputum 556 can be chemically dynamic, anoxic, and extremely reduced due to hydrogen sulfide formation.

557 MBio. 2015;6:e0767-15.

558 27. Quinn RA, Lim W, Maughan H, Conrad D, Rohwer F, Whiteson L. Biogeochemical Forces 559 Shape the Composition and Physiology of Polymicrobial Communities in the Cystic Fibrosis 560 Lung. MBio. 2014;5:1-13.

561 28. Lim YW, Schmieder R, Haynes M, Furlan M, Matthews TD, Whiteson K, et al. Mechanistic 
562 Model of Rothia mucilaginosa Adaptation toward Persistence in the CF Lung, Based on a

563 Genome Reconstructed from Metagenomic Data. PLoS One. 2013;8:e64285.

564 29. Quinn RA, Whiteson K, Lim Y-W, Salamon P, Bailey B, Mienardi S, et al. A Winogradsky-

565 based culture system shows an association between microbial fermentation and cystic fibrosis

566 exacerbation. ISME J. 2015;9:1024-38.

567 30. Whiteson KL, Meinardi S, Lim YW, Schmieder R, Maughan H, Quinn R, et al. Breath gas

568 metabolites and bacterial metagenomes from cystic fibrosis airways indicate active $\mathrm{pH}$ neutral 2 ,

569 3-butanedione fermentation. ISME J. 2014;8:1247.

570 31. Quinn RA, Whiteson K, Lim YW, Zhao J, Conrad D, LiPuma JJ, et al. Ecological

571 networking of cystic fibrosis lung infections. NPJ biofilms microbiomes. 2016;2:4.

572 32. Flynn JM, Niccum D, Dunitz JM, Hunter RC. Evidence and Role for Bacterial Mucin

573 Degradation in Cystic Fibrosis Airway Disease. 2016;:1-21.

574 33. Acosta N, Heirali A, Somayaji R, Surette MG, Workentine ML, Sibley CD, et al. Sputum 575 microbiota is predictive of long-term clinical outcomes in young adults with cystic fibrosis.

576 Thorax. 2018;73:1016-25.

577 34. Conrad DJ, Bailey BA, Hardie JA, Bakke PS, Eagan TML, Aarli BB. Median regression 578 spline modeling of longitudinal FEV1 measurements in cystic fibrosis (CF) and chronic 579 obstructive pulmonary disease (COPD) patients. PLoS One. 2017;12:e0190061.

580 35. Cobián Güemes AG, Lim YW, Quinn RA, Conrad DJ, Benler S, Maughan H, et al. Cystic 581 Fibrosis Rapid Response: Translating Multi-omics Data into Clinically Relevant Information. 582 MBio. 2019;10.

583 36. Phan J, Meinardi S, Barletta B, Blake DR, Whiteson K. Stable isotope profiles reveal active 584 production of VOCs from human-associated microbes. J Breath Res. 2017;11:17101. 
585 37. Phan J, Gallagher T, Oliver A, England WE, Whiteson K. Fermentation products in the 586 cystic fibrosis airways induce aggregation and dormancy-associated expression profiles in a CF 587 clinical isolate of Pseudomonas aeruginosa. FEMS Microbiol Lett. 2018; March:1-11.

588 38. Gao B, Gallagher T, Zhang Y, Elbadawi-Sidhu M, Lai Z, Fiehn O, et al. Tracking 589 polymicrobial metabolism in cystic fibrosis airways: Pseudomonas aeruginosa metabolism and 590 physiology are influenced by Rothia mucilaginosa-derived metabolites. mSphere. $591 \quad 2018 ; 3: \mathrm{e} 00151-18$.

592 39. Root RK, Waldvogel F, Corey L, Stamm WE. Clinical infectious diseases: a practical 593 approach. Oxford University Press, USA; 1999.

594 40. Smith EE, Buckley DG, Wu Z, Saenphimmachak C, Hoffman LR, D’Argenio DA, et al. 595 Genetic adaptation by Pseudomonas aeruginosa to the airways of cystic fibrosis patients. Proc 596 Natl Acad Sci. 2006;103:8487-92.

597 41. Adamowicz EM, Flynn J, Hunter RC, Harcombe WR. Cross-feeding modulates antibiotic 598 tolerance in bacterial communities. ISME J. 2018;:2723-35. doi:10.1038/s41396-018-0212-z. 599 42. Climo MW, Israel DS, Wong ES, Williams D, Coudron P, Markowitz SM. Hospital-wide 600 restriction of clindamycin: effect on the incidence of Clostridium difficile-associated diarrhea 601 and cost. Ann Intern Med. 1998;128 12_Part_1:989-95.

602 43. Hankinson JL, Odencrantz JR, Fedan KB. Spirometric reference values from a sample of the 603 general US population. Am J Respir Crit Care Med. 1999;159:179-87.

604 44. Schluchter MD, Konstan MW, Drumm ML, Yankaskas JR, Knowles MR. Classifying 605 Severity of Cystic Fibrosis Lung Disease Using Longitudinal Pulmonary Function Data. Am J 606 Respir Crit Care Med. 2006;174:780-6. doi:10.1164/rccm.200512-1919OC.

607 45. Sriramulu DD, Lünsdorf H, Lam JS, Römling U. Microcolony formation: a novel biofilm 
608 model of Pseudomonas aeruginosa for the cystic fibrosis lung. J Med Microbiol. 2005;54:66760976.

610 46. Palmer KL, Aye LM, Whiteley M. Nutritional cues control Pseudomonas aeruginosa

611 multicellular behavior in cystic fibrosis sputum. J Bacteriol. 2007;189:8079-87.

612 47. Fung C, Naughton S, Turnbull L, Tingpej P, Rose B, Arthur J, et al. Gene expression of

613 Pseudomonas aeruginosa in a mucin-containing synthetic growth medium mimicking cystic

614 fibrosis lung sputum. J Med Microbiol. 2010.

615 48. Cantu VA, Sadural J, Edwards R. PRINSEQ++, a multi-threaded tool for fast and efficient 616 quality control and preprocessing of sequencing datasets. PeerJ Prepr. 2019;7:e27553v1.

617 49. Ponstingl H, Ning Z. SMALT-a new mapper for DNA sequencing reads. F1000 Posters.

$618 \quad 2010 ; 1: 313$.

619 50. Bankevich A, Nurk S, Antipov D, Gurevich AA, Dvorkin M, Kulikov AS, et al. SPAdes: a 620 new genome assembly algorithm and its applications to single-cell sequencing. J Comput Biol. $621 \quad 2012 ; 19: 455-77$.

622 51. Smart KF, Aggio RBM, Van Houtte JR, Villas-Bôas SG. Analytical platform for 623 metabolome analysis of microbial cells using methyl chloroformate derivatization followed by 624 gas chromatography-mass spectrometry. Nat Protoc. 2010;5:1709.

625 52. Eren AM, Esen ÖC, Quince C, Vineis JH, Morrison HG, Sogin ML, et al. Anvi’o: an 626 advanced analysis and visualization platform for 'omics data. PeerJ. 2015;3:e1319.

627 53. Cobián Güemes AG, Youle M, Cantú VA, Felts B, Nulton J, Rohwer F. Viruses as winners 628 in the game of life. Annu Rev Virol. 2016;3:197-214.

629 54. Li H. Microbiome, metagenomics, and high-dimensional compositional data analysis. Annu 630 Rev Stat Its Appl. 2015;2:73-94. 
631 55. Thompson J, Johansen R, Dunbar J, Munsky B. Machine learning to predict microbial

632 community functions: An analysis of dissolved organic carbon from litter decomposition.

633 BioRxiv. 2019;:599704.

634 


\section{Figures}

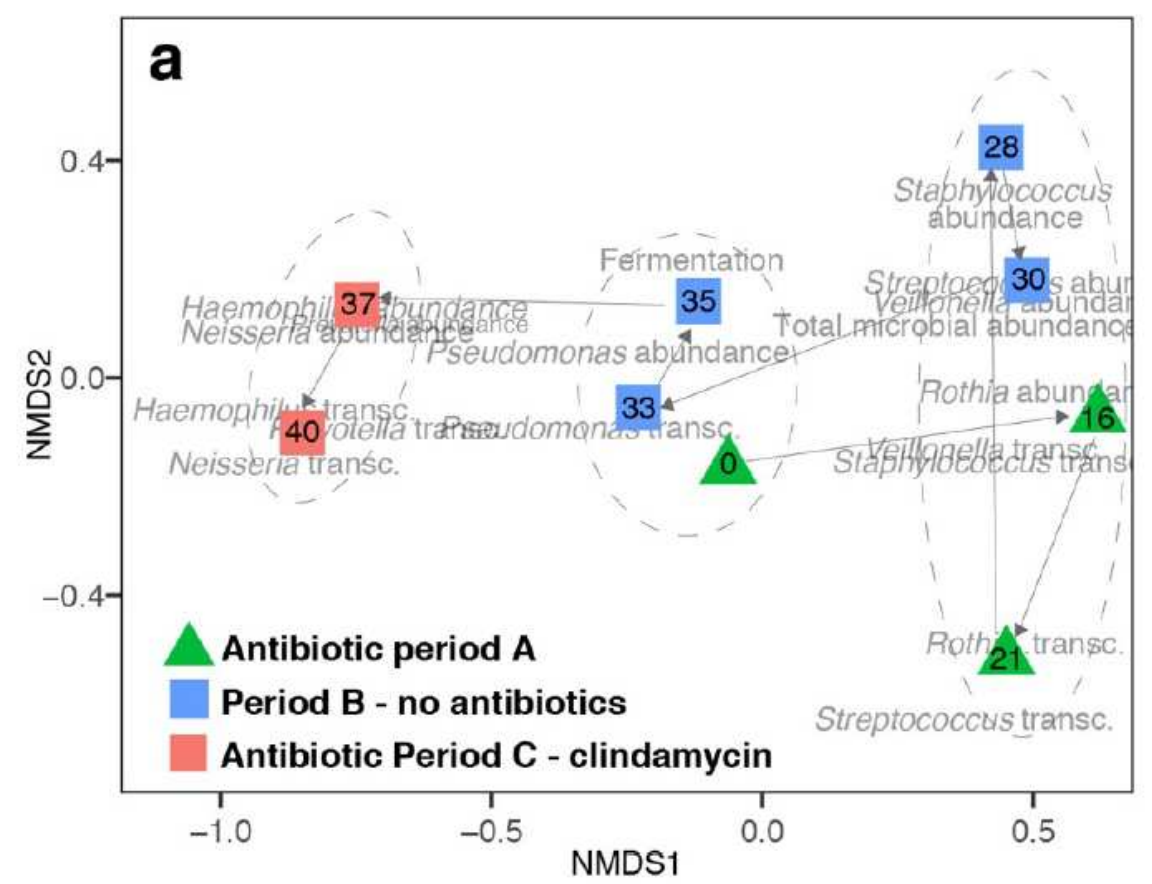

C

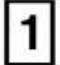

Facultative anaerobes, efficient

mucus degradation

\section{Streptococcus} Rothia

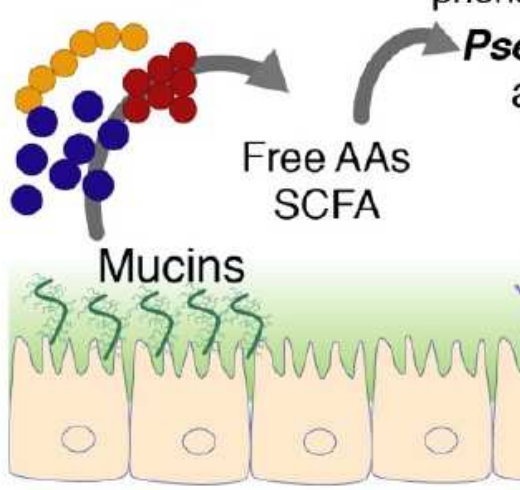

b Random forest important variables

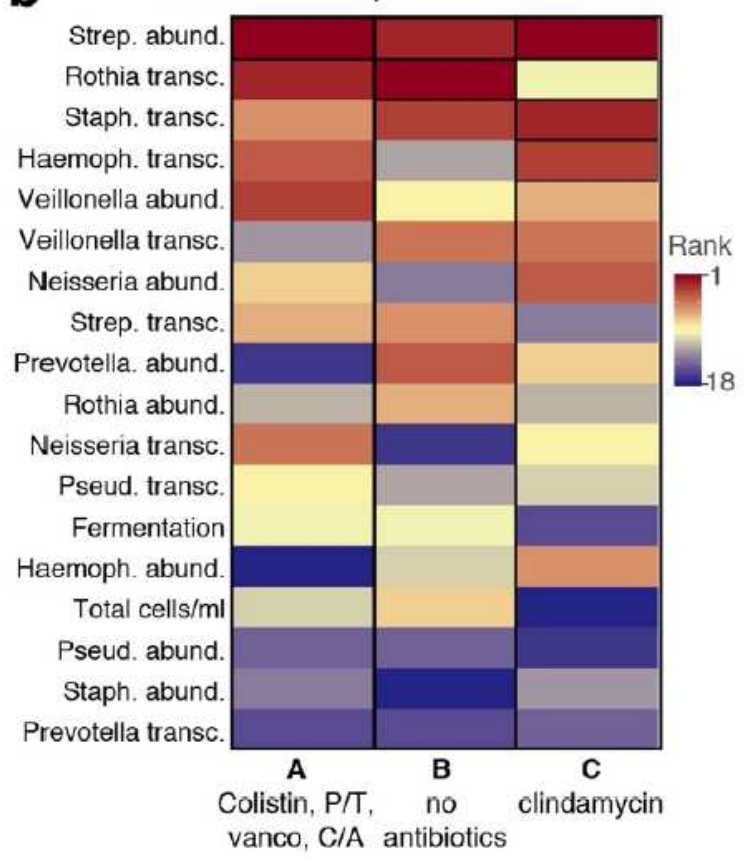

3 Anaerobic growth, mucus degradation, cross-feeding

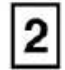
mucus-degrading, phenazine-producing Streptococcus
Inefficient

Veillonella Prevotella Staphylococcus Rothia anaerobic biofilm

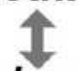

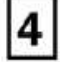

\section{Clindamycin}

Suppression of anaerobic, mucus-degrading bacteria leads to Pseudomonas starvation

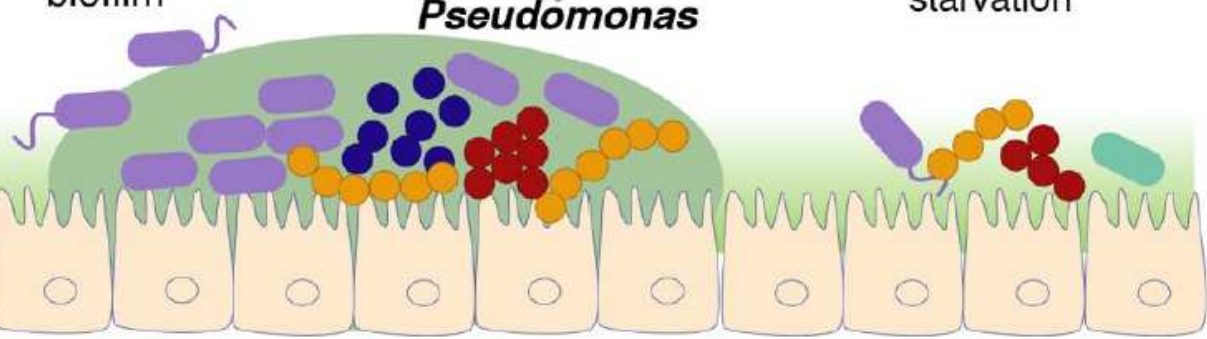

Figure 1

Microbial community succession. a) Non-metric multidimensional scaling (NMDS) of sputum samples over time. The variables used as input for the NMDS were WinCF fermentation, total microbial abundances, genera-specific microbial abundances, and genera specific transcriptomic activity. Symbols are color-coded by antibiotic treatment periods (green: A - colistin, vancomycin, ceftazidime-avibactam, and piperacillin-tazobactam; blue: B - no antibiotics; pink: C - clindamycin), and numbers inside the 
symbols indicate days since hospital admission event when Rapid Response was initiated. The dotted ellipses indicate health status groups supported by an unsupervised random forest analysis followed by clustering using Ward distances (out of bag error $=11.1 \%$ ). b) Important variables differentiating treatment periods in a classification random forest analysis supervised by treatments. The ranks surrounded by a black box indicate variables with p-values less than 0.05 in the permutational test. c) Conceptual model of succession events in patient CF146: 1- The mucosal surfaces are colonized by facultative anaerobes, including Streptococcus and Rothia, capable of efficient mucus degradation producing free-amino acids and short-chain fatty acids (SCFA, i.e., propionate, acetate, butyrate, and butanediol). 2- The free amino acids and SCFAs open a niche for the growth of Pseudomonas, which degrades mucins poorly. Pseudomonas grows forming an anaerobic biofilm, and produces phenazines. 3- The Pseudomonas biofilm facilitates the growth of anaerobes, including obligate anaerobes such as Veillonella and Prevotella. There is an overall growth of the whole microbial community, with Pseudomonas benefitting from the metabolic products from anaerobes. 4- The onset of clindamycin treatment suppresses the growth of gram positive anaerobes, such as Streptococcus and Staphylococcus. The suppression of mucus degrading bacteria removes the main nutritional source for Pseudomonas, leading to an overall decrease in microbial abundances. Period A corresponds to 28 days of hospitalization when the patient was treatment with colistin, vancomycin, piperacillin-tazobactam, and ceftazidime avibactam; in period Period B the patient was released and was off antibiotics; Period $C$ is the clindamycin treatment.

a Microbial abundances per $\mathrm{ml}$ of sputum at genus level

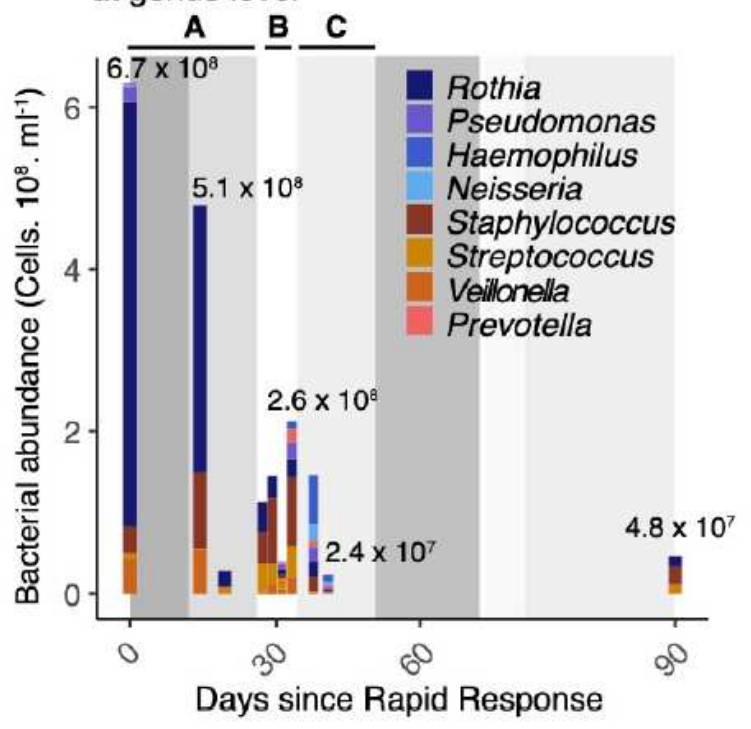

b Transcript abundace per $\mathrm{ml}$ of sputum at species level

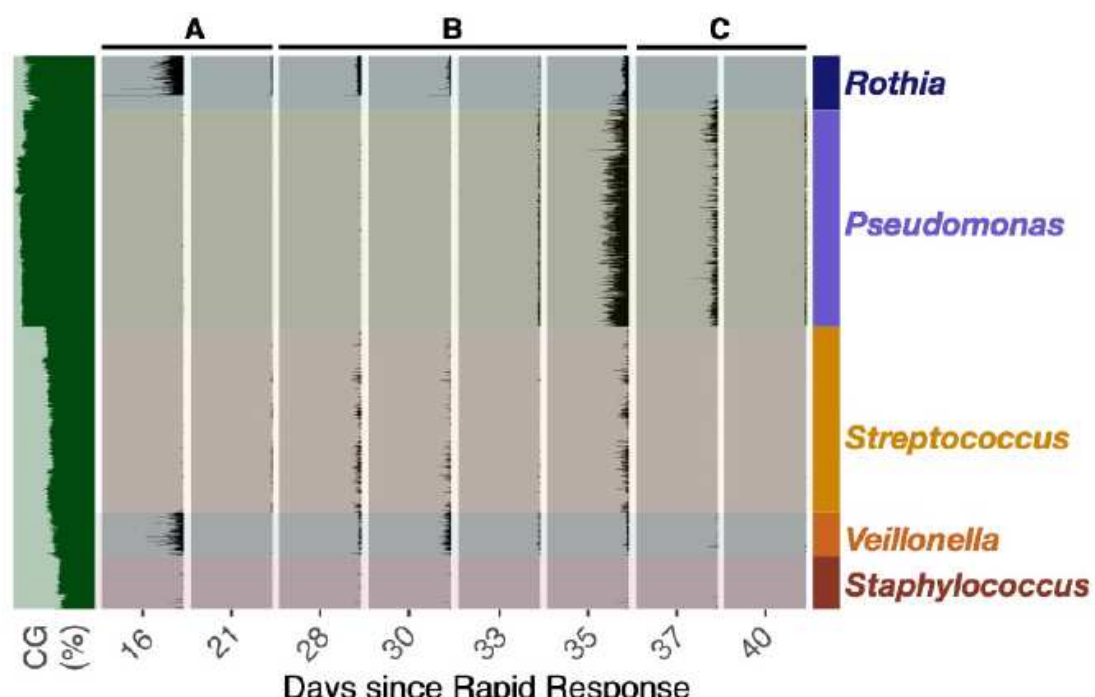

Days since Rapid Response

\section{Figure 2}

a) Changes in abundance of most abundant bacterial genera in the sputum from patient CF146. Absolute abundances (in cell counts per $\mathrm{ml}$ of sputum) were obtained by multiplying the fractional abundance from metagenomes by the total cell counts from epifluorescence microscopy. b) Recruitment of metatranscriptomic reads to contigs obtained from sequencing the genomes of clinical bacterial isolates. 
The height of each peak in the plot denotes mean coverage of a contig divided by overall sample mean coverage and scaled to the absolute microbial abundances per $\mathrm{ml}$ of sputum sample. As these are draft and not complete genomes, the position of each contig along the $y$ axis is defined by k-mer similarity $(k=$ 4) rather than position in the genome. The letters $A, B$ and $C$ within the plot area indicate the different antibiotic treatment periods.

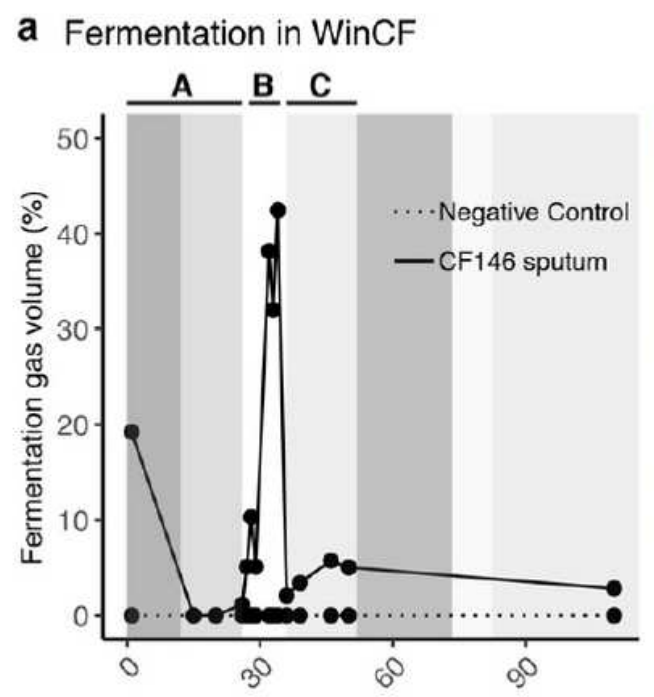

b Oxic layer in WinCF

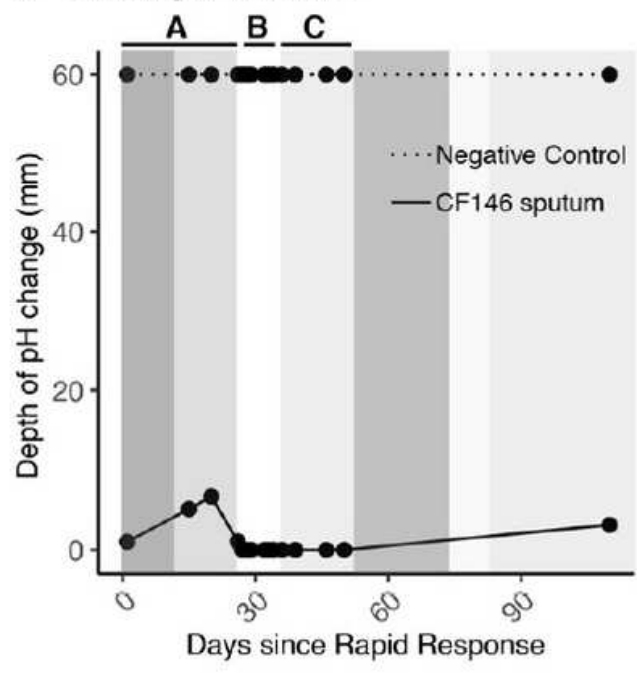

C GC metabolomics in sputum

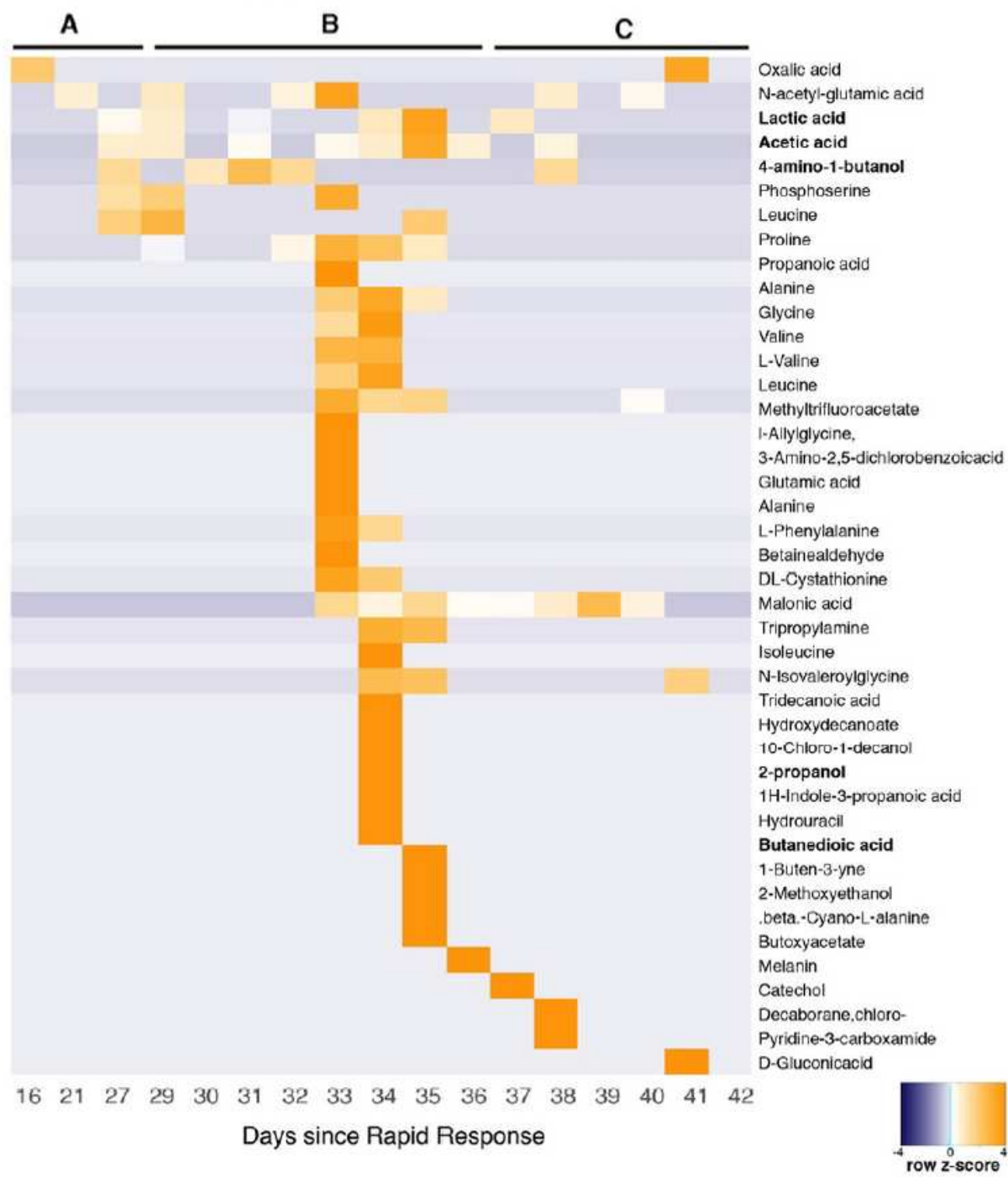

\section{Figure 3}

Fermentation in the sputum of patient CF146. a) production of fermentation gas in in WinCF capillary tubes inoculated with sputum microbial communities in artificial sputum media; $b$ ) depth of $\mathrm{pH}$ change in WinCF capillary tubes. The depth of tube where the $\mathrm{pH}$ changes corresponds to the transition from the oxic to anoxic environment and is analogous to the oxygen penetrance in the mucus plugs in the lung [29]. The letters A, B and C within the plot area indicate the different antibiotic treatment periods. 


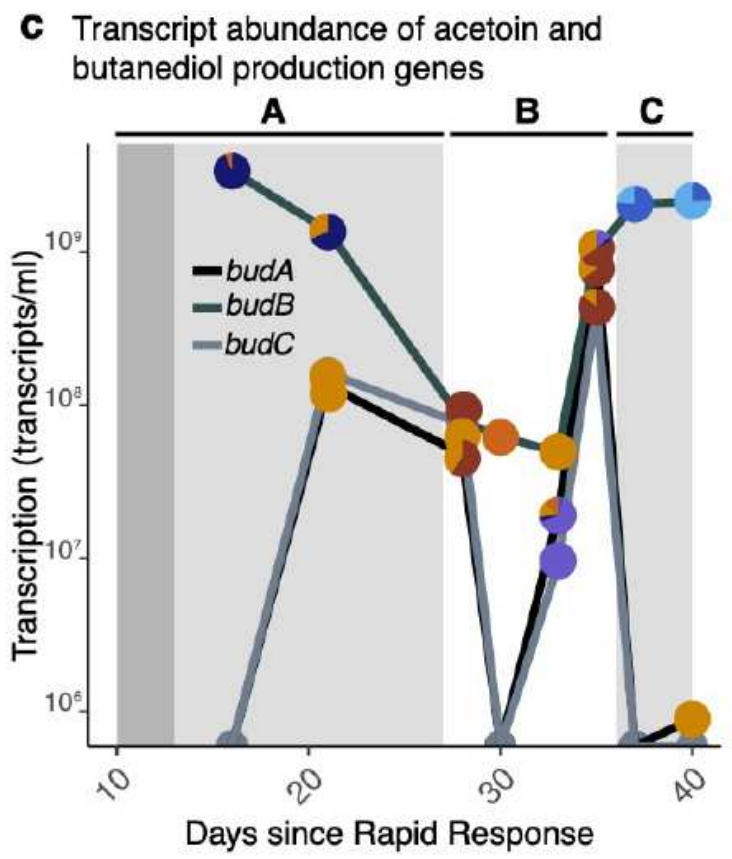

d Transcrip abundance of butanediol consumption and phenazine synthesis genes

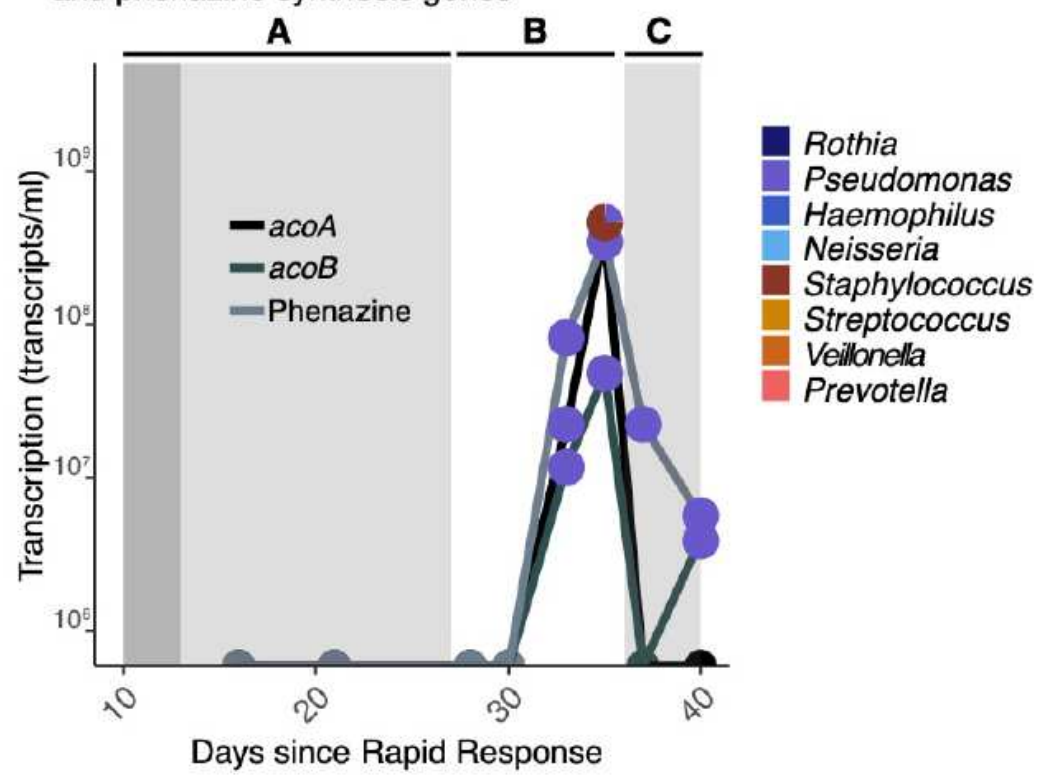

Figure 4

Microbial transcriptome dynamics. c) Abundance of transcripts involved in the pathway of acetoin and butanediol production (budA, budB, and budC). Each point in the plot is divided by the taxonomic assignment of the transcripts. The transcript abundance per $\mathrm{ml}$ ( $\mathrm{y}$ axis) was calculated as the product of the number of transcripts per $\mathrm{Kb}$ of a given taxon and the total number of $\mathrm{Kbs}$ of a that same taxon, calculated from the absolute abundances and genome sizes. d) Abundance of transcripts involved in the pathway of acetoin and butanediol consumption ( $\mathrm{aco} A$ and $\mathrm{acoB}$ ) and phenazine production (phzABCDEFG, shown as a sum of all transcripts in this gene cluster). Each point in the plot is divided by the taxonomic assignment of the transcripts. The transcript abundance per $\mathrm{ml}$ ( $\mathrm{y}$ axis) was calculated as in $4 \mathrm{~d}$.

\section{Supplementary Files}

This is a list of supplementary files associated with this preprint. Click to download.

- CF146alldataforstats.csv

- CF146Rscriptsforpublication.R

- CF146fev.csv 